\title{
Für oder wider die Struktur oder die Illusion der Abstinenz auf dem Weg zu einer gruppenanalytischen bzw. psychoanalytischen Identität
}

\author{
Sonja Wuhrmann (Zürich)
}

Zusammenfassung: Gäbe es das Seminar für Gruppenanalyse Zürich (SGAZ) ohne das PSZ? Die Frage ist müssig, aber sicher ist, dass das SGAZ, so wie es sich heute präsentiert, ohne das PSZ nicht denkbar ist. Zwar bietet es einen curricularen Ausbildungsgang mit Abschluss an, verfügt als Verein über entsprechende Strukturen mit Statuten, Dinge also, die dem Grundgedanken des PSZ widersprechen, und trotzdem sind die Ähnlichkeiten unübersehbar. Das liegt aber nicht nur daran, dass das SGAZ trotz seiner Strukturen basisdemokratisch organisiert ist. Meine Überlegungen gehen dahin, dass sich der gemeinsam geteilte Hintergrund der Psychoanalyse bestimmend auf die Institutionsdynamik auswirkt und mit der Etablierung der beruflichen Identität eines jeden Einzelnen verbunden ist. An strukturellen Debatten wird nicht nur Organisationales abgehandelt, sondern es geht immer auch um die Entwicklung einer gruppenanalytischen bzw. psychoanalytischen Identität. Dieser Thematik werde ich im Folgenden nachgehen und aufzeigen, dass psychoanalytische Organisationen - und dazu zählt auch die Gruppenanalyse - die organisationale und die individualpsychologische Ebene ständig vermischen müssen, weil die Etablierung einer beruflichen Identität über den Ablösungsprozess von den Vorbildern verläuft. Lehnt die Organisation eine Hierarchisierung ab, so verläuft die Ablösung über informelle Strukturen, die sich durch die verschiedenen Generationen zwangsläufig etablieren. Um diese Vermischung deutlich zu machen, muss der Begriff der Abstinenz in die Auseinandersetzung miteinbezogen werden. Ich plädiere für die Schaffung eines Raumes, in dem kontinuierlich an dieser Thematik gearbeitet werden kann. Die Institutionsgrossgruppe ermöglicht dann nicht nur eine organisatorische Selbstreflexion, die zur Organisationsentwicklung beiträgt, sondern fördert auch die Identitätsbildung der Einzelnen.

Schlüsselwörter: psychoanalytische und gruppenanalytische Identität, Generationenkonflikt -Abstinenz, organisationale Struktur, Institution/Organisation. 
Der einzige formal verbindliche Text über Organisation und Leitidee des PSZ ist jeweils auf den ersten vier Seiten des Semesterprogramms nachzulesen: "Durch seine innere Organisation versucht das Seminar, einen Raum zu schaffen, in dem Psychoanalyse in ihrer Konflikthaftigkeit und Widersprüchlichkeit vermittelt und erfahren werden kann. Diese Organisation ist nicht dazu da Wissen zu verwalten, sondern ihr Ziel ist es, den nie abschliessbaren Prozess zu fördern, der Wissen stets aufs Neue erzeugt.» Und weiter: Der «Besonderheit psychoanalytischer Kompetenz kann nicht durch einen vorstrukturierten Studiengang mit abschliessbarer Diplomierung entsprochen werden» (2007: 2-3). Auf der manifesten Ebene stellt sich das Psychoanalytische Seminar als ein Ort dar, der eine Ausbildung zur Psychoanalytikerin bzw. zum -analytiker anbietet und dabei die Überzeugung pflegt, dass die psychoanalytische Identität nicht durch einen formalen Abschluss erworben werden kann, sondern als ein nicht abschliessbarer Entwicklungsprozess innerpsychisch etabliert werden muss. Untrennbar mit dieser Überzeugung verbunden ist die Ablehnung hierarchischer Strukturen, sodass es am Seminar nur einen Status gibt, den der Teilnehmenden.

Da aber eine Organisation nicht statisch, sondern dynamisch ist, versteht es sich von selbst, dass Veränderungen nicht nur aktiv initiiert, sondern auch passiv ausgehalten werden müssen. Eingebettet in Kultur und Gesellschaft, wirken diese auf die Organisation so ein, dass sie sich, ob sie das will oder nicht, verändert, wie z. B. durch die Anzahl der Mitglieder, den Status, den die Psychoanalyse in der Gesellschaft hat usw. Seit 1982 wird die oben beschriebene Grundüberzeugung unverändert aufrechterhalten, obwohl oder gerade weil der Druck der Aussen- auf die Innenwelt des Seminars stetig zunimmt. Das bedeutet, dass Veränderungen als Anpassungsleistungen erbracht werden müssen. Eine solche Anpassungsleistung ist mit der Etablierung des «Blüemlimodells» gelungen. Dieses Modell erlaubte es, sich den Veränderungen der Zeit anzupassen, ohne den Grundgedanken aufzugeben, und mit einem überarbeiteten Flyer, quasi nebenbei, noch eine curriculare Weiterbildung einzuführen. Der Hinweis auf die Ausbildung und die psychoanalytische Weiterbildung in Erwachsenen- und Jugendlichen-Psychotherapie ist im Semesterprogramm in kursiver Schrift geschrieben und deutet damit auf die ambivalente Haltung hin. Der Grundgedanke des PSZ impliziert aber, dass eine strukturierte Ausbildung mit Abschluss eine Weiterentwicklung verhindert und zur Erstarrung führen muss.

Aber stimmt das? Betrachtet man nämlich die latente Ebene, so hat sich über die Jahre sozusagen ein zweites «Blüemli» entwickelt. Die Offenheit, die das PSZ seit den Anfängen pflegt, ermöglichte es, dass sich rund ums PSZ Gruppen bilden 
konnten, die sich wie einzelne Blätter ausnehmen. Sie werden freundlich toleriert, aber nicht wirklich wahrgenommen und auch nicht als zugehörig betrachtet. Man kann sich aber fragen, ob sie nicht eine notwendige Funktion für das Seminar erfüllen, indem sie die Produktion von Unbewusstheit (Erdheim 1984) gewährleisten und damit zugehöriger sind, als dies auf der manifesten Ebene wahrgenommen wird. Unter Ausschluss der Seminaröffentlichkeit sozusagen werden nämlich eine Art Probehandlungen möglich. Verpöntes kann ausprobiert und gelebt werden, wie z. B. sich als Verein zu statuieren, Strukturen zu etablieren und sogar einen Abschluss zu kreieren.

Einer dieser Vereine ist das Seminar für Gruppenanalyse Zürich. Das SGAZ nimmt insofern eine spezielle Position ein, als es als Untermieterin, seine Aus- und Weiterbildung in den Räumlichkeiten des Seminars anbietet, dasselbe Sekretariat beansprucht und auch die Adresse teilt.

Das Seminar für Gruppenanalyse macht nun alles das, was für das PSZ undenkbar ist, und versteht sich trotzdem als basisdemokratisch verwaltete Organisation. So ist zum Beispiel das SGAZ ein Verein mit entsprechenden Statuten, der eine curriculare Weiterbildung mit Abschluss anbietet und in Deutschland längst als offizielle Weiterbildungsstätte anerkannt ist. Obwohl es Strukturen kennt, international anerkannt und integriert ist, sich also bestehenden Normen anpasst, hat es sich nicht unterworfen, sondern konnte seine Eigenständigkeit bis heute bewahren. Das tönt idyllisch. Es scheint, als hätte das SGAZ den «Fünfer und das Weggli», einen Abschluss, der keine Sanktion ist, eine Struktur, die Orientierung ermöglicht, aber nicht hierarchisch ist und trotz Anpassung an internationale Normen eigenständig bleibt.

Aber eben, den «Fünfer und das Weggli» gibt es nicht. Weder gibt es weniger Konflikte, noch tut sich das gruppenanalytische Seminar mitVeränderungen leichter oder initiiert sie aktiver. Denn, so meine Hypothese, ob hierarchisch strukturiert oder basisdemokratisch organisiert, mit Abschluss oder ohne, die Konflikte innerhalb der psycho- und gruppenanalytischen Institutionen verlaufen ähnlich. Sie unterscheiden sich höchstens in der Heftigkeit. Denn bei den Auseinandersetzungen um organisatorische Strukturen geht es nicht nur um die Entwicklung und Veränderung der Organisation, sondern immer auch um die Entwicklung einer beruflichen Identität für jeden Einzelnen. Das heisst, dass es sich bei der Auseinandersetzung um strukturelle Themen jeweils auch um die Frage der gruppen- oder psychoanalytischen Identität handelt. Betrachtet man z. B. die «Journal»-Ausgaben und Titel von Tagungen seit Bestehen des PSZ, so wird deutlich, wie sehr sich dieses immer wieder mit sich selber befasst. Unzählige Autorinnen und Autoren haben sich Gedanken gemacht 
und Artikel zur Geschichte des PSZ verfasst. Eigentlich wäre alles gesagt, Deutungen ausgesprochen und mehrfach wiederholt, aber trotzdem ändert sich nur wenig, so als handelte es sich um eine unendliche Analyse. Nur, das ist nicht nur am psychoanalytischen Seminar so. Es ist auffallend, wie sehr psychoanalytische und gruppenanalytische Institute mit sich ringen. Aber genau das deutet darauf hin, dass es sich bei dieser Thematik nicht nur um eine institutionelle, sondern auch um eine individualpsychologische Auseinandersetzung mit der analytischen Identität und damit auch mit der Bedeutung der Abstinenz handelt. Denn der Versuch, in der Gruppenselbsterfahrung oder in der Einzel-Analyse einen abstinenten Raum aufrecht zu erhalten, in dem Übertragungen sich entfalten, bearbeitet und aufgelöst werden können, muss scheitern. Es gibt keinen abstinenten Raum, wenn sich AnalytikerInnen und AnalysandInnen, SupervisorInnen und SupervisandInnen in derselben Organisation bewegen. Diese werden ja zuerst einmal als machtvolle Instanzen idealisiert, und der Weg zur Etablierung der beruflichen Identität kann nur über die Loslösung und die Entidealisierung dieser Vorbilder laufen. Dass es gleichzeitig auch eine Schwierigkeit für Analytikerinnen und Analytiker selber bedeuten kann, wenn sie ihre eigene Entidealisierung vorantreiben müssen und sich am selben Ort wie ihre AnalysandInnen bewegen, scheint nachvollziehbar. Ich bin der Überzeugung, dass es eine Illusion ist, zu glauben, dass sich Übertragungen, die sich in diesem Rahmen entwickeln, jemals vollständig auflösen können. Und das gilt unabhängig davon, ob Analytikerin bzw. Analytiker und Analysandin bzw. Analysand versuchen, sich während des analytischen Prozesses am Seminar aus demWeg zu gehen. Diese Realität macht deutlich, was es bedeutet, eine analytische Identität zu entwickeln. Rolf Haubl (2005) zieht zur Erklärung dieser Schwierigkeiten das Modell von Jürgen Habermas über Moralentwicklung und Ich-Identität (1976) heran, das eine Bewegung über drei Identitätsformationen beschreibt, einer präkonventionellen, einer konventionellen und einer post-konventionellen. In der prä-konventionellen Formation sind wir mit wichtigen persönlichen Vorbildern identifiziert, wie z. B. der Gruppenlehranalytikerin oder dem Einzel-Analytiker. Eine konventionelle Identitätsformation liegt vor, wenn die Identifikation mit den Theorien und Normen, die diese Vorbilder vertreten, möglich ist, wie z. B. die Identifikation mit bestimmten Schulen oder Ausbildungsinstituten, die diese vertreten. In der postkonventionellen Formation schliesslich ist die Entpersonalisierung vollzogen und die Identifikation verläuft über theoretische Inhalte und deren Auseinandersetzung. Die Vermutung liegt nahe, dass wir oft konventionell identifiziert bleiben.

Vor diesem Hintergrund betrachtet muss sich am SGAZ und am PSZ zwangsläufig eine vergleichbare Institutionsdynamik zeigen, obwohl das eine Strukturen 
kennt und das andere nicht. Als Teilnehmerin beider Institute werde ich mich auf das SGAZ beschränken, um die Bedeutung des abstinenten Raumes und der damit verbundenen Entwicklung einer beruflichen Identität aufzuzeigen, wobei deutlich wird, dass eine Trennung zwischen Einzel- und Gruppenanalyse kaum möglich ist. Da aber die Ausbildung in Form von viertägigen Sequenzen stattfindet und die Übertragungsprozesse auch die horizontale Ebene, die der peergroup, umfassen, kann man sich diesen Prozessen am SGAZ weniger entziehen. Während die Auflösung der Idealisierung in der Einzelanalyse der dyadischen Konstellation verhaftet bleibt, kommt in der Gruppenanalyse ein zusätzliches Moment hinzu. Die geschwisterlichen Übertragungsbeziehungen komplizieren die Entwicklung zusätzlich, umso mehr, wenn die Organisationsform nicht hierarchisch strukturiert ist, weil die Heftigkeit der geschwisterlichen Rivalität dadurch provoziert werden kann.

Aber zuerst zur formalen Organisation des SGAZ. Im Laufe der ersten zehn Jahre hat es sich Schritt für Schritt entwickelt, auf der formalen Ebene Strukturen eingeführt, einen curricularen Abschluss erarbeitet und dabei die basisdemokratische Verwaltung so weit als möglich beibehalten. So kennt die Vereinsform mit einem Vorstand kein Präsidium, sondern eine Kontaktperson nach aussen. Das Organisationskomitee ist für Fragen der Ausbildungsteilnehmer während der Sequenzen zuständig und das Ausbildungsgremium zeichnet für die Inhalte der Ausbildung und für den Abschluss verantwortlich. Entsprechend der basisdemokratischen Organisation hat der Vorstand und das Ausbildungsgremium also fast keine Kompetenzen, weil die Entscheidungshoheit bei der Mitgliederversammlung liegt.

Eine Besonderheit des Gruppenanalytischen Seminars ist es, dass rund die Hälfte der Mitglieder aus Deutschland und einige wenige aus Österreich kommen. Der kulturelle Einfluss wirkt sich bestimmend auf die Entwicklung des Seminars aus, das in der Zwischenzeit rund 140 Mitglieder zählt, wovon 40 in Ausbildung sind. Die grosse Anzahl der deutschen Teilnehmenden zwang relativ bald zu einer strukturierten Ausbildung mitAbschluss, weil eine berufs- und gesundheitspolitische Notwendigkeit der Anerkennung bestand. Dem fiel die Selbstdeklaration zum Opfer. Das SGAZ stellt heute ein Zertifikat aus und verleiht den Titel Gruppenanalytikerin bzw. Gruppenanalytiker SGAZ. Dies hat sich aber als fruchtbar für die Entwicklung des Seminars erwiesen. Bildlich gesprochen, wurden wir SchweizerInnen zwar von hinten angestossen und mussten widerwillig Strukturen einführen, aber gleichzeitig gelang es - mit dem PSZ im Rücken - an der Basisdemokratie festzuhalten. Für diejenigen, die aus Deutschland kommen, ist gerade die geringe Hierarchisierung 
attraktiv. Bis 2006 stand das Seminar unter der Schirmherrschaft der Group Analytic Society in London, da aber das Thema der Anerkennung auch in der Schweiz bald abgeschlossen sein dürfte, konnte diese aufgehoben werden.

Den zu Beginn aus England bzw. vom Londoner Gruppenanalytischen Institut kommenden GruppenanalytikerInnen, die bei der Entwicklung der Organisation mithalfen und zu Beginn auch an den OK- und später an den Mitgliederversammlungen teilnahmen, blieb letztlich das Verständnis für unsere Organisation verschlossen, und schon bald drohten sie mit dem Entzug der Anerkennung durch die Group Analytic Society in London, weil sie nicht verstehen konnten, dass wir nicht einfach alles von ihnen Geforderte übernehmen wollten, und nur mit Mühe akzeptierten, dass diejenigen, welche sie als Teilnehmende in der Selbsterfahrungsgruppe hatten, am Businesslunch mit ihnen über Honorar und Steuern stritten. Eine Verletzung der Abstinenzregel war das und eigentlich unhaltbar, aber es ging ja nicht anders, da die meisten Teilnehmenden noch in Ausbildung waren. Das bedeutete für die Institutionsdynamik, dass Generationenkonflikte und Machtkämpfe auch über die Auseinandersetzung mit organisationalen Themen ausgetragen werden konnten. Mit der steigenden Anzahl von Mitgliedern, die die Ausbildung beendet hatten, veränderte sich aber zwangsläufig etwas, denn jetzt gab es Mitglieder in Ausbildung und Mitglieder nach Ausbildung. Um den Ausbildungsteilnehmenden einen möglichst freien Übertragungsraum gewährleisten zu können, wurden die Funktionen in den Gremien zunehmend durch Mitglieder nach Ausbildung besetzt. Durch die Ausbildungsorganisation der slow-open Gruppen bedeutete dies, dass sich «Gruppengeschwister» in unterschiedlichen Funktionen begegneten. So kann sich jemand noch in der Ausbildung befinden, während ein anderes Gruppenmitglied die Ausbildung abschliesst. Zurzeit fordern die Statuten, dass eines der fünf Vorstandsmitglieder noch in Ausbildung ist. Auch die Trainer bzw. Gruppenlehranalytiker, die nach wie vor alle von aussen bzw. anderen Instituten kommen, können sich heute auf ihre Funktion als Gruppenleitende, SupervisorInnen und TheoriereferentInnen zurückziehen, um die gruppenanalytische Selbsterfahrung möglichst frei von Institutionsbelangen zu halten. Dass damit aber eine informelle Hierarchisierung eingeführt wurde, dessen war man sich nicht bewusst.

Denn diese Veränderung, die sich nicht aktiv, sondern durch die Zeit - das SGAZ ist 25-jährig - und die steigende Anzahl der Mitglieder ergeben hat, hat Implikationen, mit denen die Organisation auf der dynamischen Ebene ringt. Denn neben der oben erwähnten informellen Ebene wurde mit der Einführung eines Abschlusses im Grunde die erste und bis jetzt einzige formale hierarchische Struktur eingeführt. Ins Ausbildungsgremium kommt nämlich nur, wer über einen 
Abschluss verfügt, den längst nicht alle machen. Das bedeutet eine entsprechend geringe Anzahl von Personen, die für dieses Gremium überhaupt wählbar sind. Da die Trainer nicht mehr in Organisationsentscheidungen eingebunden sind, sind sie diejenigen, die ein Stück befreit sind vom institutionellen Übertragungsraum. Dafür ist der Raum frei für die geschwisterlichen Übertragungen, die dann auch ausserhalb der Kleingruppen virulent werden müssen, wenn sichtbar wird, dass nicht mehr alle gleich sind. Das öffnet Projektionen Tür und Tor, weil der Ort der Begegnung fast nur noch die Mitgliederversammlung ist. Übertragungsebene und organisationale Ebene vermischen sich ständig, weil Abstinenz nicht mehr möglich ist.

Nun ist aber zu fragen, ob es eine wirkliche Abstinenz in der Ausbildung überhaupt geben kann, oder ob wir uns nicht von einer Illusion verabschieden müssen. Das, was wir nämlich unseren KlientInnen gegenüber als abstinente Haltung vertreten, ist in einer Ausbildung, ob zur Gruppenanalytikerin oder zur Psychoanalytikerin, nicht aufrecht zu erhalten.

Gruppenanalytische Institute sind keine Biotope, in denen sich Übertragungen frei entfalten können, sie gleichen eher Aquarien. Abgegrenzt nach allen Seiten, schwimmt man im immer gleichen Becken herum. Die Mitglieder haben meist einen ähnlichen beruflichen Hintergrund und sie verfolgen einen Zweck mit der Teilnahme an der Selbsterfahrung. Diese ist Herzstück einer Ausbildung und nicht primärTherapie-mindestens machen wir uns das vor-, und schon bald versuchen wir, theoretisch zu verstehen, was wir erleben. Fantasien und Realitäten mischen sich, weil geschwisterliche Beziehungen einen grossen Raum einnehmen und nicht auf die Kleingruppe beschränkt werden können, sondern gerade durch die basisdemokratische Organisation institutionell zum Tragen kommen. Dies alles kompliziert dann die freie Entfaltung der Übertragungen und auch deren mögliche Auflösung, wenn wir uns vormachen, dass eine abstinente Haltung impliziert, dass sich die Teilnehmenden von allen Verantwortlichkeiten fernhalten sollten, was in einer Ausbildung unmöglich ist.

In seinem Beitrag am Heidelberger Symposium «Abstinenz: Grenze oder Schranke in der gruppenanalytischen Ausbildung» hat Werner Knauss diese Frage aus der Sicht des Lehrgruppenanalytikers aufgeworfen. Er greift zuerst auf Freuds Konzeptualisierung zurück, der den Begriff der Abstinenz von «die Kur muss ...» zu die «(...) Kur soll soweit als möglich in der Entbehrung - Abstinenz-durchgeführt werden» relativiert (Knauss 1994: 396). Später hat er die negative Formulierung dieses Ratschlags bedauert, indem er anmerkt, dass er die positive Seite der Beziehung zum Analysanden zwar praktiziert aber nicht theoretisch ausgeführt 
hat. Den Widerspruch zwischen der methodisch begründeten Abstinenzregel und seiner eigenen freizügigen therapeutischen Praxis erklärt Freud durch die Zweiteilung des Übertragungsbegriffs, indem er die neurotische Übertragung von einer unanstössigen, der realen Beziehung, unterscheidet. Paula Heimann (1950), die die kommunikative Bedeutung der Gegenübertragung im analytischen Prozess erstmals konzeptualisiert, relativiert damit auch die geforderte Neutralität und Abstinenz, weil sie die in der Analytikerin auftauchenden Gefühle und Impulse als das wichtigste Arbeitsinstrument betrachtet. Diese sind dahingehend zu untersuchen, ob sie der eigenen Konflikthaftigkeit entspringen oder ob sie Hinweise auf unbewusste Prozesse in den Analysanden oder in der Übertragungsbeziehung enthalten. Zudem hat die Analytikerin zu prüfen, ob das, was sie sagt oder tut, im Interesse der Analysanden oder im eigenen Interesse geschieht.

Was bedeutet nun für Knauss Abstinenzin der Ausbildung? Er plädiert dafür, auf verschiedenen Ebenen als Lehrgruppenanalytiker mit AusbildungsteilnehmerInnen zusammenzuarbeiten. DieVerantwortung lokalisiert er ganz klar beim Lehranalytiker bzw. beim Gruppenleiter. In Arbeitsgruppen begegnet er GruppenteilnehmerInnen kollegial und arbeitet sachbezogen ohne falsche Hemmungen, da der ausseranalytische Kontakt kein privater ist, sondern im Rahmen des Instituts stattfindet. Gleichwohl wird jede Begegnung ausserhalb der Gruppe innerhalb der Gruppe analysiert und bearbeitet. Er ist der Überzeugung, dass es in der Ausbildungssituation nicht darum geht, sich mit dem Analytiker zu identifizieren, sondern mit seiner analytischen Funktion. Diese Funktion kann aber nur dann deutlich werden, wenn die AusbildungsteilnehmerInnen die Möglichkeit haben, den Analytiker in seiner analytischen Funktion, also im Prozess des Analysierens, zu erleben. Das heisst sichtbar zu machen, wie er mit bestimmten Regeln in einer bestimmten Situation, in einem spezifischen Moment der analytischen Übertragungs-GegenübertragungsKonstellation jeweils modifiziert umgehen kann. Cremerius (1984) nennt dies den operationalen Gebrauch der Abstinenz. Knauss ist der Überzeugung, dass Verbote, die damit begründet werden, die AusbildungsteilnehmerInnen vor Gefühlsverwirrungen zu schützen, mehr mit der Angst der LehranalytikerInnen vor Entidealisierung zu tun haben als mit einem Schutz der AusbildungsteilnehmerInnen.

OttoF.Kernberg(1998) hatsichmitdemThema derpsychoanalytischenAusbildung und deren Instituten eingehend und in kritischerWeise auseinandergesetzt. Immer wieder betont er die Schwierigkeiten und die Gefahr des Kreativitätsverlustes, die sich aus den spezifischen Abhängigkeiten durch die Ausbildung ergeben. Buchstäblich alle psychoanalytischen Institute seien gekennzeichnet durch Verfolgungsfantasien und unrealistische Idealisierungen als Folge der Anonymität der Lehranalytiker. 
Kurt Buchinger (1993) hat in seinem Psyche-Artikel «Zur Organisation psychoanalytischer Institutionen» wichtige Fragen aufgegriffen, die die Geschichte der psychoanalytischen Bewegung begleiten und die auch für die gruppenanalytische Bewegung gelten.

Buchinger macht auf zwei Fehler aufmerksam, denen psychoanalytische Institutionen immer wieder unterliegen. Zum einen wird die Unterscheidung zwischen Psychodynamik und Dynamik in Institutionen ständig vermischt, und zum anderen wird eine organisierte Berufsgruppe immer wieder mit der Familie verwechselt. Er fordert, nicht nur in Personen und Beziehungen zu denken, sondern vielmehr auch in Strukturen denken und handeln zu lernen.

Im Sinne eines Lernprozesses geht es darum, neben der spezifisch psychoanalytischen Selbstreflexion eine organisatorische Selbstreflexion auszubilden. Nach Buchinger geht es darum, «die Ausbildung von Organisationsbewusstsein, also die Entwicklung einer Fähigkeit in Strukturen und Prozessen organisatorischer Art zu denken und zu diagnostizieren, nicht bloss in Kategorien von Personen und ihren Beziehungen» (1997:55). Anknüpfend an einen Begriff von Norbert Elias (1983) betont er, dass organisatorische Selbstreflexion ein hohes Mass an «Distanzierungsfähigkeitin-Beteiligtsein» (1997: 54) verlange.

Bei der Lektüre von Buchingers Artikel wurde mir klar, dass wir im SGAZ, wie in allen psychoanalytischen Instituten, immer wieder versucht sind, in familialen Strukturen zu denken, aber längst über den Punkt hinaus gelangt sind, an dem dies der Realität entsprechen könnte. Unterstützt wird dieser Gedanke durch den Artikel «Anachronizität und Unbewusstheit» von Mario Erdheim (1995). Er bezieht sich darin auf den Widerspruch zwischen Familie und Kultur. Der Ablösungsprozess in der Adoleszenz konzentriert sich um einen Konflikt herum, um den Antagonismus zwischen Familie und Kultur. In dem Mass, in dem sich die Ablösung von der Familie vollzieht, bilden Familie und Kultur zwei nicht mehr vereinbare Systeme. Gerade dies eröffnet dem Individuum aber neue kreative Perspektiven, die den Ablösungsprozess unterstützen. Erdheim verweist nun darauf, dass Institutionen, welche diesen Antagonismus verleugnen, die Tendenz haben, in ihrem Kern die Struktur der unbewussten Familie herzustellen. Dann entsteht aber eine Diskrepanz zwischen Funktion und Symbolik, welche dieWahrnehmung der Realität erschwert. Die Folge ist, dass sich die Vergangenheit in der Gegenwart immer wieder durchzusetzen vermag und so Veränderung verhindert, wenn nicht gar verunmöglicht. Erdheim betont auch, dass gerade psychoanalytische Institutionen in dieser Hinsicht gefährdet sind. 
Das Denken in familialen Strukturen führt Buchinger darauf zurück, dass die Psychoanalyse aus einer Bewegung entstand, die personenbezogen war durch Freud und durch die Psychoanalyse an sich. Der zentrale Gegenstand ihrer Theorie ist die Psychodynamik der Persönlichkeit, in ihrer Entwicklung im familialen Beziehungssystem, und die Technik soll einen strukturellen Eingriff in das psychische System eines Menschen ermöglichen. Wenn man in seiner Arbeit die eigene Person als Arbeitsinstrument einsetzen und ständig in Frage stellen muss, so scheint es ausserdem nur zu verständlich, dass man sich eine Institution wünscht, in der man, so wie man ist, angenommen wird - wie in einer Familie, auch wenn diese dann immer wieder enttäuscht. Da unsere Arbeit immer zutiefst subjektiv und personenbezogen ist, ist auch verständlich, dass wir dazu neigen, die entstehenden Konflikte vor diesem Hintergrund zu interpretieren und zu personalisieren.

Das Arbeitsinstrument der PsychoanalytikerInnen, so Buchinger, kann zur destruktivenWaffe werden, indem Konflikte in Institutionen durch neurotische Konflikte erklärt werden. So werden organisatorische Sachverhalte auf psychische reduziert, was die Probleme nicht löst, sondern bestenfalls einfach nicht verändert.

Auch die Gruppenanalyse ist aus einer Bewegung entstanden, auch sie personenbezogen durch Foulkes und bestimmt durch ein familienanaloges System - die Selbsterfahrungsgruppe -, ihr zentrales Arbeitsinstrument. Ihr Spezifikum liegt noch darin, dass das «Mutterhaus», das Londoner Institut, sehr präsent ist, indem die Ausbildung an vielen Instituten eng durch dieses begleitet wurde. Die Versuchung also, als «Töchter» und «Söhne» endlich erwachsen zu werden oder als «Geschwister» Rivalitäten auszutragen, wird nochmals potenziert und läuft damit Gefahr, mit der Entwicklung der Organisation verwechselt zu werden.

Buchinger betont, dass komplexe Ansätze wie die Psychoanalyse in allen ihren Teilen störanfällig sind und gerade darum in ihrer Reproduktion - in der Ausbildung-besonderen Schutz verdienten. Dieser ist aber nicht dadurch zu bewerkstelligen, dass man einzelne Teile voneinander isoliert, sondern sie im Gegenteil einer gemeinsamen Reflexion zugänglich macht. "Eine gemeinsame, institutionell abgesicherte, in der Öffentlichkeit der Vereine durchgeführte Selbstreflexion des Zusammenhangs zwischen den einzelnen Teilen der Ausbildung ist, so unser Postulat, als ein Teil der Ausbildung einzuführen. Sie ist in ihrer Bedeutung nicht unter der Lehranalyse anzusetzen» (1997: 62).

Voraussetzung einer kontinuierlichen institutionellen Selbstreflexion ist allerdings auch ein ungehemmter, sachbezogener Umgang der Ausbildungsteilnehmenden und der Mitglieder untereinander. Und damit kehre ich wieder zum Begriff der Abstinenz zurück. Ich bin der Überzeugung, dass es sinnvoll wäre, sich damit aus- 
einanderzusetzen, was wir als Mitglieder in und nach der Ausbildung unter unserer Abstinenz verstehen. Das gilt besonders für die Gruppenanalyse, weil sich das Übertragungsgeschehen nicht auf die beschränken, welche die Gruppen leiten, sondern eben auch alle Mitglieder der Gruppe umfasst. Gruppenmitglieder und Gruppenleiter sind eine Achse, Gruppenmitglieder untereinander bilden die andere. Das gilt aber auch für das PSZ, in dem die Bedeutung der peergroup zwangsläufig weniger aktiv reflektiert wird und durch die Dyade in der Einzelanalyse Gefahr läuft, die Abstinenz zwar formal einzuhalten, was aber damit einhergehen kann, dass die Bearbeitung der Übertragungsauflösung auf den Praxisraum beschränkt bleibt. Wenn die Verantwortung an den Seminaren wirklich gemeinsam getragen werden soll, dann kann es nicht darum gehen, die Mitglieder in Ausbildung vor ihrerVerantwortlichkeit zu schützen. Abstinenz könnte dann als Grenze zwischen Psychodynamik und Organisationsdynamik verstanden werden. Der Ort, um diese Vermischungen immer wieder zu bearbeiten, ist die Institutionsgrossgruppe. Nur dann besteht die Möglichkeit, dass wiederkehrende Konflikte bearbeitet werden können und so den Weg zurWeiterentwicklung der Organisation frei und auch den Weg zur beruflichen Identität erträglicher machen. Denn Veränderung geschieht. Diese in einem Setting zu reflektieren, in der beide Ebenen sichtbar sein dürfen, die psychodynamische und die organisationale, entspricht eigentlich einer Notwendigkeit.

\section{Literatur}

Buchinger, K. (1993):Zur Organisation Psychoanalytischer Institutionen: Psychoanalyse geht nicht ohne, Psychoanalyse geht nicht mit. Oder doch? In: Psyche, 1: 31-70.

Buchinger, K. (1997): Supervision in Organisationen. Heidelberg: Verlag Auer,

Elias, N. (1990): Engagement und Distanzierung. Frankfurt: Suhrkamp Verlag (1. Aufl. 1983).

Erdheim, M. (1984): Die gesellschaftliche Produktion von Unbewusstheit. Eine Einführung in den ethnopsychoanalytischen Prozess. Frankfurt: Suhrkamp Verlag (1. Aufl. 1982).

Erdheim, M. (1995): Anachronizität und Unbewusstheit.Zum Problem der Reproduktion vergangener gesellschaftlicher Wirklichkeiten. In: Gruppenanalyse, 5/1: S. 9-26.

Freud, S. (1991): Bemerkungen über die Übertragungsliebe, Frankfurt: Fischer Verlag, GWX, (Erstpublikation 1915). 
Freud, .S. (1982):Wege der psychoanalytischen Therapie, Studienausgabe, GWXII. Frankfurt: Fischer Taschenbuch Verlag (Erstpublikation 1919).

Haubl, R. (2005): Reinheit und Gefährdung - Gruppenanalyse auf dem Weg zur Realitätstauglichkeit. In: Gruppentherapie und Gruppendynamik, 41: S. 267-285.

Habermas, J. (1976): Zur Rekonstruktion des Historischen Materialismus. Frankfurt: Suhrkamp Verlag.

Heimann, P. (1950): Bemerkungen zur Gegenübertragung. In: Psyche, 1964, 18: S. $483-493$.

Knauss, W. (1994): Abstinenz: Grenze oder Schranke in der Gruppenanalytischen Ausbildung. In: 9th ESGA, Boundaries and Barriers, Heidelberg: Mattes Verlag: 394-401.

Kernberg, O.F. (1988): Zerstörung der Psychoanalyse im Ausbildungssystem. In: Psyche, 3: S. 199-213. 